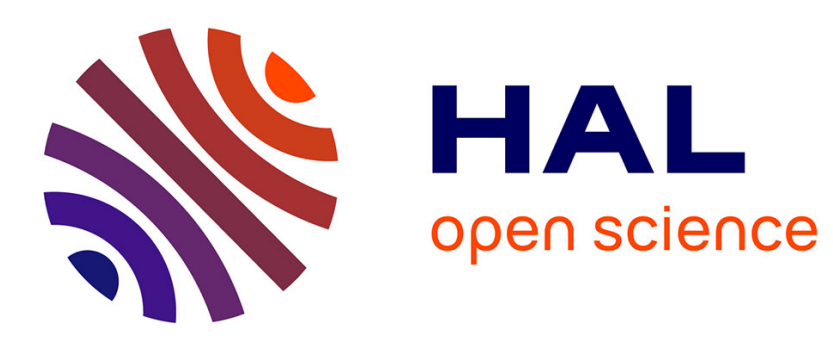

\title{
Infinités de la puissance rayonnée par un dipole dans un milieu ionisé puissance irréversible-puissance réversible J.P. Lafon
}

\section{To cite this version:}

J.P. Lafon. Infinités de la puissance rayonnée par un dipole dans un milieu ionisé puissance irréversible-puissance réversible. Revue de Physique Appliquée, 1968, 3 (3), pp.216-222. 10.1051/rphysap:0196800303021600 . jpa-00242850

\section{HAL Id: jpa-00242850 https://hal.science/jpa-00242850}

Submitted on 1 Jan 1968

HAL is a multi-disciplinary open access archive for the deposit and dissemination of scientific research documents, whether they are published or not. The documents may come from teaching and research institutions in France or abroad, or from public or private research centers.
L'archive ouverte pluridisciplinaire HAL, est destinée au dépôt et à la diffusion de documents scientifiques de niveau recherche, publiés ou non, émanant des établissements d'enseignement et de recherche français ou étrangers, des laboratoires publics ou privés. 


\title{
INFINITÉS DE LA PUISSANCE RAYONNÉE PAR UN DIPOLE DANS UN MILIEU IONISÉ PUISSANGE IRRÉVERSIBLE-PUISSANGE RÉVERSIBLE
}

\author{
Par J. P. LAFON, \\ Groupes d'Astronomie spatiale, Observatoire de Paris, Meudon. \\ (Reçu le 12 juillet 1967.)
}

\begin{abstract}
Résumé. - On étudie le tenseur de Green dans le cas d'un plasma sans collision. D'une part, on considère qu'il existe des relations entre $\mathbf{E}_{\text {out }}$ champ sortant et $\mathbf{E}_{\text {in }}$ champ entrant auxquelles correspondent des relations entre $\mathscr{G}_{\text {in }}$ tenseur entrant et $\mathscr{G}_{\text {out }}$ tenseur sortant. Les cas des plasmas froids homogènes en présence d'un champ magnétique fini (cas biaxe) ou infini (cas uniaxe) sont plus particulièrement étudiés. Le calcul est effectué in extenso pour le terme $G_{\mathrm{zz}}$ dans le second cas. Les résultats et les raisonnements sont confrontés avec ceux de Lee et Papas (cf. "Radio Science " [1]) et ceux de Staras [2] que l'on conteste sur plusieurs points. On discute la régularité des solutions d'une équation d'onde homogène, ni contredite par Staras ("Radio Science " [2]) ni démontrée par Lee et Papas. On discute de l'introduction de collisions dans le calcul de $P$. Enfin, l'on montre que le partage effectué par Lee et Papas de la puissance rayonnée en $P_{\text {réversible }}$ et $P_{\text {irréversible conduit toujours à : }}$

$$
P_{\text {irréversible }}=P_{\text {totale }} ; \quad P_{\text {réversible }}=0 \text {. }
$$
\end{abstract}

\begin{abstract}
We study the Green's tensor for the case of a collisionless plasma. First, we consider the relations which exist between $\mathbf{E}_{\text {out }}$, the "outgoing field", and $\mathbf{E}_{\text {in }}$, the "ingoing field". These relations correspond to similar expressions for the "outgoing" and "ingoing" tensors $\mathscr{G}_{\text {out }}$ and $\mathscr{G}_{\text {in }}$. The case of a cold, homogeneous plasma in the presence of a finite (biaxial case) or infinite (uniaxial case) magnetic field is given particular attention. A complete calculation of $G_{\mathrm{zz}}$ is done for the uniaxial case. The results and reasoning are compared with those of Lee and Papas (1965 and 1966) and of Staras (1966), and several points of disagreement are found. We discuss the regularity of the solutions of the homogeneous wave equation, which has been neither contradicted by Staras nor demonstrated by Lee and Papas. We also discuss the introduction of collisions into the calculations of the radiated power $P$. Finally, we show that the separation of the radiated power into $P_{\text {reversible }}$ and $P_{\text {irreversible }}$ (as done by Lee and Papas) always leads to :
\end{abstract}

$$
P_{\text {irreversible }}=P_{\text {total }} ; \quad P_{\text {reversible }}=0 .
$$

Dans plusieurs articles cités en référence, Lee et Papas ont voulu montrer que dans un plasma sans collisions les valeurs infinies prises par la puissance rayonnée, corrélatives aux valeurs infinies prises par l'indice de réfraction dans certaines directions (1), n'étaient en fait dues qu'à un mauvais calcul de cette puissance. Selon ces auteurs, l'on devrait dédoubler l'expression classique $P$ en deux parties, l'une supportant sans changement un renversement du temps, champ magnétique et grandeurs associées, $P_{\text {rev }}\left({ }^{2}\right)$ à négliger, l'autre représentant la vraie énergie rayonnée

(1) Surfaces d'indices hyperboliques.

(2) $P$ réversible. antisymétrique par rapport à cette transformation, $P_{\text {irr }}\left({ }^{3}\right)$.

Weil et Walsh [3] ont déjà répondu en discutant le point de vue physique de la question.

Nous nous proposons d'en étudier l'aspect mathématique.

Il existe enfin un commentaire de Staras [2]. Mais ses résultats sont incertains car il applique des propriétés de symétrie à des intégrales soumises à conditions de rayonnement dans leur définition, ce qui est contestable.

C'est pourquoi nous allons reprendre l'étude au

(3) $P$ irréversible. 
point de départ dans le cas des plasmas froids, homogènes, en présence d'un champ magnétique fini (cas biaxe) ou infini (uniaxe), cas dans lesquels ces divers auteurs se sont successivement placés.

Nous démontrons que dans ces cas respectifs on a toujours les relations :

$$
\begin{array}{c|c}
\text { Cas biaxe } & \text { Cas uniaxe } \\
\mathbf{E}_{\mathrm{in}}\left(-t,-\mathbf{B}_{\mathbf{0}}\right)=\mathbf{E}_{\mathrm{out}}^{*}\left(t, \mathbf{B}_{\mathbf{0}}\right) & \mathbf{E}_{\mathrm{in}}(-t)=\mathbf{E}_{\mathrm{out}}^{*}(+t) \\
P_{\mathrm{rev}}=0 & P_{\mathrm{irr}}=P .
\end{array}
$$

Nous discuterons enfin en conclusion les diverses incidences de ces assertions.

I. Cas du champ magnétique fini. - Nous nous plaçons toujours en régime permanent; les champs et courants varient dans le temps de façon sinusoïdale à la pulsation $\omega\left(\mathrm{e}^{j \omega t}\right)$.

La fonction de Green du milieu considéré vaut alors :

$$
\mathscr{G}\left(\mathbf{r}-\mathbf{r}^{\prime}\right)=\int_{\text {espace }} \frac{j \omega \mu_{0}}{8 \pi^{3}} \lambda^{-1} \mathrm{e}^{-j \mathbf{k}\left(r-r^{\prime}\right)} \mathrm{d} \mathbf{k}
$$

avec :

$$
\begin{gathered}
\lambda^{-1}=\boldsymbol{\Lambda} / D \quad \boldsymbol{\Lambda}=k^{2} \mathbf{k k}-k_{0}^{2} k^{2} \mathscr{L}+k_{0}^{4} \mathscr{E} \\
D=k_{0}^{2}\left\{k^{4}\left(\varepsilon_{1} \sin ^{2} \theta+\varepsilon_{3} \cos ^{2} \theta\right)-k_{0}^{2} k^{2} \times\left[\left(\varepsilon_{1}^{2}-\varepsilon_{2}^{2}\right) \sin ^{2} \theta\right.\right. \\
\left.\left.+\varepsilon_{1} \varepsilon_{3}\left(\cos ^{2} \theta+1\right)\right]+k_{0}^{4} \varepsilon_{3}\left(\varepsilon_{1}^{2}-\varepsilon_{2}^{2}\right)\right\} . \quad(\mathrm{I} .2)
\end{gathered}
$$

Pour des expressions de $\mathscr{E}$ et $\mathscr{L}$, cf. Kogelnik [4]. Elles sont des matrices hermitiques comme l'est par conséquent le polynôme matriciel $\boldsymbol{\Lambda}$. Avec les notations de la figure 1, on peut écrire (cf. développements

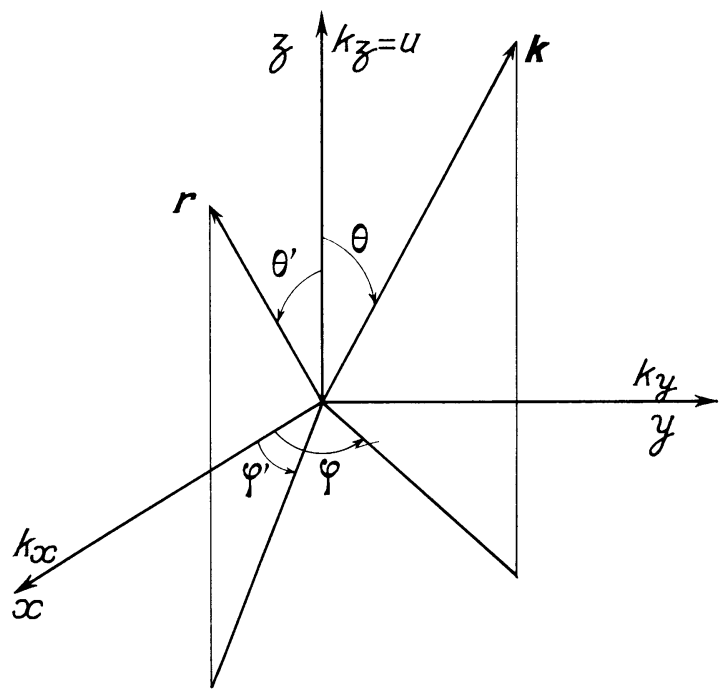

FIG. 1. dans « Conditions de rayonnement et d'antirayonnement » [6]) :

$G(\mathbf{r})=j \omega \mu_{0} \Gamma(\mathbf{r})$

$$
=\int_{\theta=0}^{\pi / 2} \int_{\varphi=0}^{2 \pi} \sin \theta \mathrm{d} \theta \mathrm{d} \varphi \frac{\Lambda\left(\theta, \varphi, j \frac{\partial}{\partial \rho}, \omega\right)}{k_{0}^{2}\left(\varepsilon_{1} \sin ^{2} \theta+\varepsilon_{3} \cos ^{2} \theta\right)}
$$

$$
\frac{j \omega \mu_{0}}{8 \pi^{3}} \frac{\partial^{2}}{\partial \rho^{2}} \int_{-\infty}^{+\infty} \frac{\mathrm{e}^{-j k \rho}}{\Delta(k, \omega)} \mathrm{d} k
$$

$\boldsymbol{\Lambda}\left(\theta, \varphi, j \frac{\partial}{\partial \rho}, \omega\right)$ est le polynôme $\boldsymbol{\Lambda}(\theta, \varphi, k, \omega) \operatorname{de}(\mathrm{I} .2)$ où l'on a substitué à $k, j \frac{\partial}{\partial \rho}$ :

$$
\begin{aligned}
& \text { k. } \mathbf{r}=k \rho(\theta, \varphi) \\
& \quad=k r\left(\sin \theta \sin \theta^{\prime} \cos \left(\varphi-\varphi^{\prime}\right)+\cos \theta \cos \theta^{\prime}\right)
\end{aligned}
$$

et :

$$
\begin{aligned}
& D=k_{0}^{2}\left(\varepsilon_{1} \sin ^{2} \theta+\varepsilon_{3} \cos ^{2} \theta\right) \Delta \\
& \Delta=\left(k^{2}-k_{1}^{2}\right)\left(k^{2}-k_{2}^{2}\right) .
\end{aligned}
$$

On sait par ailleurs que :

$$
\mathbf{E}(\mathbf{r}, t)=\int_{\text {espace }} \mathscr{G}\left(\mathbf{r}-\mathbf{r}^{\prime}\right) \mathbf{E}\left(\mathbf{r}^{\prime}, t\right) \underline{\mathrm{d} \mathbf{r}^{\prime}} .
$$

On posera encore :

$$
\Phi(\rho)=\int_{-\infty}^{+\infty} \frac{\mathrm{e}^{-j k \rho}}{\Delta(k, \omega)} \mathrm{d} k
$$

Remarque. - L'intégrale que nous avons écrite sous la forme $\Phi(\rho)$ n'a de sens, dans le cas où les pôles $k_{1}$ ou $k_{2}$ sont réels, qu'en fonction de la condition de rayonnement, c'est-à-dire que $\int_{-\infty}^{+\infty}$ signifie qu'on intègre sur un axe réel déformé comme il convient. On définit d'ailleurs ainsi deux expressions suivant les déformations adoptées $\Phi_{\text {in }}$ et $\Phi_{\text {out }}$ correspondant à $\mathscr{G}_{\text {in }}$ et $\mathscr{G}_{\text {out }}$, et par-delà à $\mathbf{E}_{\text {in }}$ et $\mathbf{E}_{\text {out }}$. Dans le cas où $k_{1}$ est imaginaire pur, l'axe réel n'a pas à être déformé et $\Phi_{\text {in }}=\Phi_{\text {out }}$ d'après leur définition, (cf. « Condition de rayonnement » [6]).

La condition de rayonnement s'applique à l'intégrale $\Phi$ qui est sommable même dans le cas où l'indice peut devenir infini, la direction en question étant négligée (la sommation double s'effectue sur une fonction presque partout définie).

Nous allons distinguer les cas où les pôles $k_{1}$ et $k_{2}$ sont réels ou imaginaires purs.

L'intégrant est $\frac{\mathrm{e}^{-j k \rho}}{\Delta(k, \omega)}$. Lorsque $k$ tend vers l'infini avec une partie du signe de $-\rho(\rho=0$ correspondant à $k$ et $r$ perpendiculaires est négligé comme ensemble de mesure nulle), $\mathrm{e}^{-j k \rho}$ tend vers zéro plus vite que toute puissance de $k$. Comme $\Delta(k, \omega)$ se comporte à l'infini en $k$ comme la puissance $4^{\text {ième }}$ de $k$, on peut dire que l'intégrale sur un demi-cercle de rayon tendant vers $+\infty$ centré à l'origine et situé dans le demi-plan $(\operatorname{Im} k)(-\rho)>0$ tend vers 0 . 


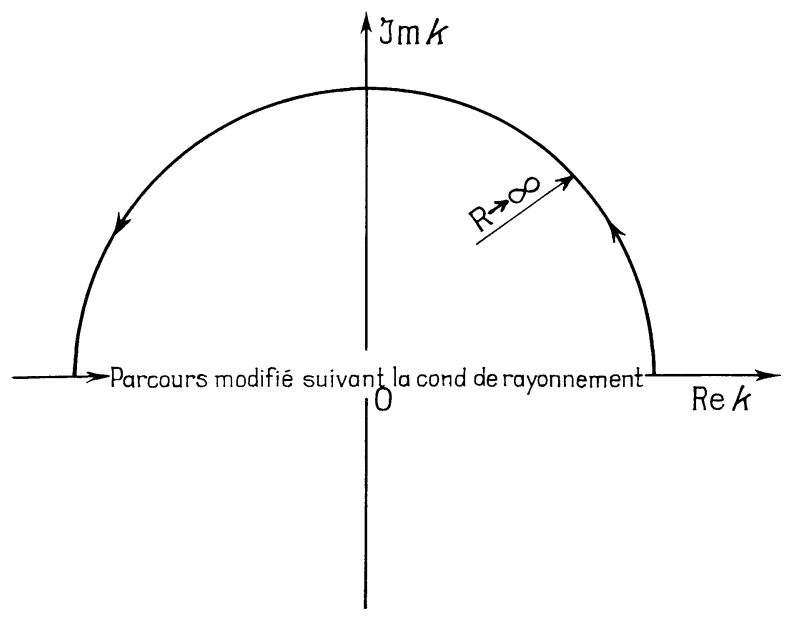

FIG. 2.

On peut donc appliquer la méthode des résidus ne utilisant un parcours semblable à celui de la figure 2.

On étudiera donc successivement les contributions d'un pôle $k_{1 \text { ou } 2}$ réel ou imaginaire pur à l'intégrale $\Phi$.

a) Contribution D'UN POLE RÉEL $k_{1}$ ET DE SON OPPOSÉ - $k_{1}$. - Quand $\omega$ prend la valeur complexe $\omega+j \eta(\eta<0)$ (ondes croissant dans le temps), le pôle $k_{1}$ prend par définition une partie imaginaire positive et évidemment $-k_{1}$ une partie imaginaire négative. Cela distingue les deux pôles opposés.

Le contour modifié passe donc au-dessous de $k_{1}$ et au-dessus de $-k_{1}$ pour définir $\Phi_{\text {out }}$.

Il passe au-dessus de $+k_{1}$ et au-dessous de $-k_{1}$ pour définir $\Phi_{\text {in }}$ (cf. fig. 3 ).
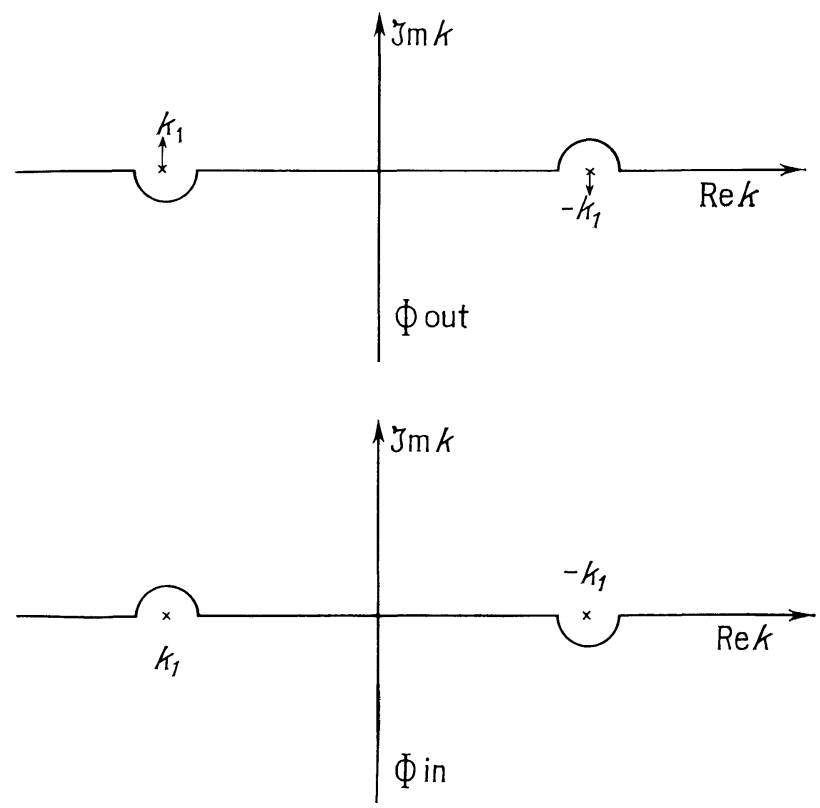

FIG. 3.
La contribution du pôle réel $k_{1}$ vaut donc, en la notant prime :

$$
\begin{gathered}
\Phi_{\text {out }}^{\prime}=\pi j \frac{\mathrm{e}^{j k_{1} \rho}}{\left(k_{1}^{2}-k_{2}^{2}\right) k_{1}} \quad \rho>0 \\
\Phi_{\text {out }}^{\prime}=\pi j \frac{\mathrm{e}^{-j k_{1} \rho}}{k_{1}\left(k_{1}^{2}-k_{2}^{2}\right)} \quad \rho<0 \\
\Phi_{\text {in }}^{\prime}=\pi j \frac{\mathrm{e}^{-j k_{1} \rho}}{\left(k_{1}^{2}-k_{2}^{2}\right)\left(-k_{1}\right)} \quad \rho>0 \\
\Phi_{\text {in }}^{\prime}=\pi j \frac{\mathrm{e}^{j k_{1} \rho}}{\left(k_{1}^{2}-k_{2}^{2}\right)\left(-k_{1}\right)} \quad \rho<0 .
\end{gathered}
$$

On voit donc que dans tous les cas :

$$
\Phi_{\text {in }}^{\prime}=\Phi_{\text {out }}^{\prime *} \text {. }
$$

b) Contribution D'Un POle imaginaire PUR $k_{2}$ ET DE SON OPPOSÉ $-k_{2}$. - On a déjà remarqué que :

$$
\Phi_{\text {in }}=\Phi_{\text {out }} \text {. }
$$

On appellera de même $k_{2}$ le pôle dont la partie imaginaire est positive (partie réelle nulle), $-k_{2}$ l'autre.

La contribution de $k_{2}$ vaut donc, en la notant encore prime :

$$
\begin{array}{ll}
\Phi_{\text {in }}=\pi j \frac{\mathrm{e}^{j k_{2} \rho}}{\left(k_{2}^{2}-k_{1}^{2}\right) k_{2}} & \rho>0 \\
\Phi_{\text {in }}^{\prime}=\pi j \frac{\mathrm{e}^{-j k_{2} \rho}}{\left(k_{2}^{2}-k_{1}^{2}\right) k_{2}} & \rho<0 .
\end{array}
$$

En général, donc $\Phi_{\text {in }}^{\prime} \neq \Phi_{\text {out }}^{\prime *}$ puisqu'il lui est égal, mais ici : $(\mathrm{I} .14 \mathrm{~b})$, on note alors que $\Phi_{\text {in }}^{\prime}$ est toujours réel car $j k_{2}$ est un réel. Donc (I.14 b) entraîne :

$$
\Phi_{\text {in }}^{\prime}=\Phi_{\text {out }}^{\prime *} \text {. }
$$

Conclusion. - On voit donc que les contributions d'un pôle sont toujours telles que pour un pôle réel (et même dans le cas présent pour un pôle imaginaire pur) :

$$
\Phi_{\text {in }}^{\prime}=\Phi_{\text {out }}^{\prime *} \text {. }
$$

On en déduit que toujours dans le cas présent :

$$
\Phi_{\text {in }}=\Phi_{\text {out }}^{*}
$$

alors qu'en général $\Phi_{\text {in }} \neq \Phi_{\text {out }}^{*}$.

Or :

$$
\mathscr{E}^{\mathscr{T} \mathrm{T}}=\mathscr{E} \quad \text { et } \quad \mathscr{L}^{* \mathrm{~T}}=\mathscr{L} \quad \text { (hermiticité) }
$$

$Y\left(-\mathbf{B}_{0}\right)=-Y\left(\mathbf{B}_{0}\right) \quad$ donc $\quad \varepsilon_{2}\left(-\mathbf{B}_{0}\right)=-\varepsilon_{2}\left(\mathbf{B}_{0}\right)$

$$
\varepsilon_{1}\left(-\mathbf{B}_{0}\right)=\varepsilon_{1}\left(\mathbf{B}_{0}\right) ; \quad \varepsilon_{3}\left(-\mathbf{B}_{0}\right)=\varepsilon_{3}\left(\mathbf{B}_{0}\right) \text {. }
$$

Donc :

$$
\begin{aligned}
& \mathscr{E}\left(\mathbf{B}_{0}\right)^{*}=\mathscr{E}\left(-\mathbf{B}_{0}\right)=\mathscr{E}^{\mathscr{T}}\left(\mathbf{B}_{\mathbf{0}}\right) \\
& \mathscr{L}\left(\mathbf{B}_{0}\right)^{*}=\mathscr{L}\left(-\mathbf{B}_{\mathbf{0}}\right)=\mathscr{L}^{\mathrm{T}}\left(\mathbf{B}_{\mathbf{0}}\right)
\end{aligned}
$$

Donc encore :

$$
\boldsymbol{\Lambda}\left(-\mathbf{B}_{\mathbf{0}}\right)=\boldsymbol{\Lambda}^{\mathrm{T}}\left(\mathbf{B}_{0}\right)=\boldsymbol{\Lambda}^{*}\left(\mathbf{B}_{0}\right)
$$

Il s'ensuit qu'avant sommation sur $\theta$ et $\varphi$ :

$\boldsymbol{\Lambda}\left(-\mathbf{B}_{\mathbf{0}}\right) \Phi_{\text {in }}=\boldsymbol{\Lambda}^{*}\left(\mathbf{B}_{\mathbf{0}}\right) \Phi_{\text {in }}=\left(\boldsymbol{\Lambda}\left(\mathbf{B}_{0}\right) \varphi_{\text {out }}\right)^{*}$. 
Donc après intégration, $k_{0}^{2}\left(\varepsilon_{1} \sin ^{2} \theta+\varepsilon_{3} \cos ^{2} \theta\right)$ étant insensible au changement de signe en $\mathbf{B}_{0}$ :

$$
\begin{aligned}
& \boldsymbol{\Gamma}_{\text {in }}\left(-\mathbf{B}_{\mathbf{0}}\right)=\boldsymbol{\Gamma}_{\text {out }}^{*}\left(\mathbf{B}_{\mathbf{0}}\right) \\
& \boldsymbol{\Gamma}_{\text {out }}\left(-\mathbf{B}_{\mathbf{0}}\right)=\boldsymbol{\Gamma}_{\text {in }}^{*}\left(\mathbf{B}_{\mathbf{0}}\right) .
\end{aligned}
$$

Avec la propriété du courant vrai supposé connu dans l'antenne :

$$
\mathbf{J}^{*}\left(-t,-\mathbf{B}_{\mathbf{0}}\right)=-\mathbf{J}\left(t, \mathbf{B}_{\mathbf{0}}\right)
$$

et la propriété de variation temporelle :

$$
\left(\mathrm{e}^{j \omega t}\right)^{*}=\mathrm{e}^{j \omega(-t)}
$$

on obtient, grâce aux formules (I.3) et (I.6) :

$$
\mathbf{E}_{\text {in }}^{*}\left(-t,-\mathbf{B}_{0}\right)=\mathbf{E}_{\text {out }}\left(t, \mathbf{B}_{0}\right) \text {. }
$$

II. Cas du champ magnétique infini (uniaxe). Les équations (I.1) et (I.2) sont à conserver avec cependant les nouvelles valeurs de $\mathscr{E}, \mathscr{L}$ et $D$ suivantes :

$$
\begin{aligned}
\mathscr{E} & =\left(\begin{array}{ccc}
1-X & 0 & 0 \\
0 & 1-X & 0 \\
0 & 0 & 1
\end{array}\right) \\
\mathscr{L} & =\left(\begin{array}{c}
n_{\alpha}^{2}+n_{\beta}^{2}+(1-X)\left(n_{\alpha}^{2}+n_{\gamma}^{2}\right) \\
(1-X) n_{\alpha} n_{\beta} \\
n_{\alpha} n_{\gamma}
\end{array}\right.
\end{aligned}
$$

$$
\left.\begin{array}{cc}
(1-X) n_{\alpha} n_{\beta} & n_{\alpha} n_{\gamma} \\
n_{\alpha}^{2}+n_{\beta}^{2}+(1-X)\left(n_{\beta}^{2}+n_{\gamma}^{2}\right) & n_{\beta} n_{\gamma} \\
n_{\beta} n_{\gamma} & 1+n_{\gamma}^{2}
\end{array}\right)
$$

k a pour composantes $k_{\mathrm{x}}=k n_{\alpha}, k_{\mathrm{y}}=k n_{\beta}, \quad k_{\mathrm{z}}=u=k n_{\gamma}$ :

$$
\begin{aligned}
D & =k_{0}^{2}\left(k^{2}-k_{0}^{2}\right)\left(k^{2}-X u^{2}+k_{0}^{2}(X-1)\right) \\
& =k_{0}^{2}\left(1-X \cos ^{2} \theta\right) \Delta .
\end{aligned}
$$

On reprend la façon d'écrire de (I.3) avec les relations (I.4), (I.5) et (I.6). Enfin, (I.7) devient :

$$
\Phi(\rho)=\int_{-\infty}^{+\infty}-\frac{\mathrm{e}^{-j k \rho}}{\Delta(k, \omega)} \mathrm{d} k
$$

avec le nouveau :

$$
\Delta=\left(k^{2}-k_{0}^{2}\right)\left(k^{2}-\frac{k_{0}^{2}(1-X)}{1-X \cos ^{2} \theta}\right)
$$

$\Delta$ est toujours un polynôme en $k$ et l'on peut appliquer la méthode des résidus en vue de calculer cette intégrale comme précédemment pour les mêmes raisons. On étudierait de même les contributions des pôles réels $\pm k_{0}$ et des pôles $\pm \sqrt{\frac{k_{0}^{2}(1-X)}{1-X \cos ^{2} \theta}}$ réels ou imaginaires purs (le cas $X=1$ n'est pas gênant car $k^{2}=0$ n'est pas pôle : $k^{2}$ figure au numérateur dans dk et a été extrait sous la forme $\partial^{2} / \partial \rho^{2}$ dans la nouvelle équation (I.3), et de la même façon on montrerait que, dans ce cas :

$$
\Phi_{\text {in }}=\Phi_{\text {out }}^{*}
$$

On remarque que, ici encore, la matrice $\mathscr{E}$ étant réelle comme $\mathscr{L}^{\text {: }}$

$$
\mathscr{E}^{* T}=\mathscr{E}^{T}=\mathscr{E}^{*}=\mathscr{E} ; \quad \mathscr{L}^{* T}=\mathscr{L}^{*}=\mathscr{L}^{T}=\mathscr{L} .
$$

Donc de même avec la nouvelle expression :

$$
\left(\Lambda \Phi_{\text {in }}\right)^{*}=\Lambda^{*} \Phi_{\text {in }}^{*}=\Lambda \Phi_{\text {out }}
$$

avant intégration, et après :

$$
\boldsymbol{\Gamma}_{\text {in }}=\boldsymbol{\Gamma}_{\text {out }}^{*}
$$

Ce qui donne avec les propriétés :

- du courant vrai,

- du facteur temps $\mathrm{e}^{j \omega t}$,

- de l'intégrale du champ (I.6) :

$$
\mathbf{E}_{\text {in }}(-t)=\mathbf{E}_{\text {out }}^{*}(t) \text {. }
$$

Cette propriété a été vérifiée sur les « éléments » à intégrer. Elle va nous permettre de calculer exactement l'intégrale calculée par Lee et Papas concernant le terme $z z$ de la fonction de Green (cf. «A further explanation of the new theory »[1]).

III. Cas uniaxe : Terme $G_{z z^{*}}$ - L'intégrale en question est :

$$
G=\frac{1}{8 \pi^{3}} \int \frac{\mathrm{e}^{j k r} k^{2} \mathrm{~d} k \sin \theta \mathrm{d} \theta \mathrm{d} \varphi}{k^{2}-k_{0}^{2}(1-X)-X k_{3}^{2}}
$$

( $G$ dans ce paragraphe a le sens que lui donnent Lee et Papas, $k_{0}$ n'est plus pôle). Notons que, sans le dire, Lee et Papas utilisent le facteur $\mathrm{e}^{-j \omega t}$ pour la variation temporelle (transformation $\omega \rightarrow \omega+j \varepsilon, \varepsilon>0$ ). Nous devons donc appliquer ainsi la condition de rayonnement.

Le calcul sera effectué en coordonnées cylindriques. C'est pourquoi nous allons étudier le pôle en $u \mathrm{du}$ dénominateur. Le cas étudié par Lee et Papas est le seul contesté, soit $X>1$; nous nous placerons dans ce cas :

$$
u_{0}= \pm \sqrt{k_{0}^{2}+\frac{t^{2}}{X-1}}
$$

$X>1$ toujours défini et réel; deux déterminations $u_{0}^{\prime}$ (signe + ) et $-u_{0}^{\prime}$. 
(Rappel :

Coordonnées cylindriques $u=k \cos \theta, \quad t=k \sin \theta$ )

$$
\frac{\mathrm{d} u_{0}^{\prime}}{\mathrm{d} \omega}=\frac{1}{2 u_{0}^{\prime}}\left(2 \frac{\omega}{c}+\frac{2 t^{2} X^{\prime}}{(X-1)^{2} \omega}\right) .
$$

La transformation $\omega \rightarrow \omega+j \eta \quad(\eta>0)$ l'affecte comme l'indique la figure 4 .

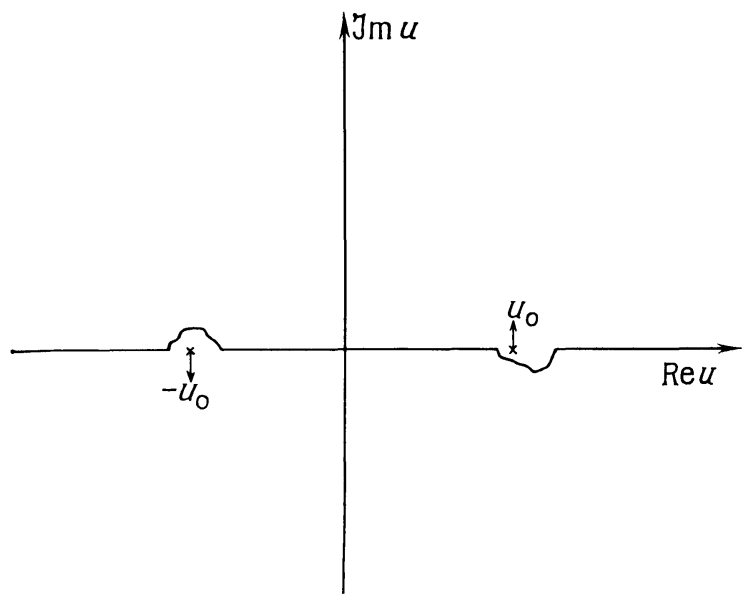

FIG. 4.

D'où les contours d'intégration indiqués sur la même figure pour $G_{\text {out }}$.

L'intégrale peut alors s'écrire après intégration sur $\varphi$ :

$$
G=\frac{1}{4 \pi^{2}(1-X)} \int_{u=-\infty}^{+\infty} \int_{t=0}^{\infty} \frac{t \mathscr{J}_{0}(t \rho) \mathrm{e}^{j z u}}{u^{2}-u_{0}^{2}} \mathrm{~d} t \mathrm{~d} u .
$$

L'intégration sera faite par la méthode des résidus comme il a été justifié dans un cas analogue au paragraphe II.

Les demi-cercles de rayon $R \infty$ seront pris au-dessus de l'axe réel pour $z>0$, au-dessous pour $z<0$ :

$$
\begin{aligned}
G_{\text {out }} & =-\frac{j}{4 \pi(X-1)} \int_{0}^{\infty} \frac{t \mathscr{J}_{0}(t \rho) \mathrm{e}^{j z u_{0}}}{u_{0}} \mathrm{~d} t z>0 \\
G_{\text {out }} & =\frac{-j}{4 \pi(X-1)} \int_{0}^{\infty} \frac{t \mathscr{J}_{0}(t \rho) \mathrm{e}^{-j z u_{0}}}{u_{0}} \mathrm{~d} t z<0
\end{aligned}
$$

Pour achever l'intégration, utilisons l'intégrale connue :

$$
\int_{0}^{\infty} \frac{X \mathscr{J}_{0}(c x) \mathrm{e}^{j a \sqrt{x^{2}+b^{2}}}}{\sqrt{x^{2}+b^{2}}} \mathrm{~d} x
$$

$\sqrt{\frac{\pi}{2}} \sqrt{b} \frac{1}{\sqrt[4]{a^{2}-c^{2}}}\left[-N_{-1 / 2}\left(b \sqrt{a^{2}-c^{2}}\right)\right.$

$$
\left.+j \mathscr{J}_{-1 / 2}\left(b \sqrt{a^{2}-c^{2}}\right)\right]
$$

si $0<c<a, \operatorname{Re} b>0$. $\sqrt{\frac{2}{\pi}} \sqrt{b}\left(c^{2}-a^{2}\right)^{-1 / 4} K_{1 / 2}\left(b \sqrt{c^{2}-a^{2}}\right)$

si $0<a<c, \operatorname{Re} b>0$

avec :

$$
\begin{gathered}
j \mathscr{J}_{-1 / 2}(z)-N_{-1 / 2}(z)=j \sqrt{2 / \pi z} \mathrm{e}^{j z} \\
K_{ \pm 1 / 2}(z)=\sqrt{\pi / 2 z} \mathrm{e}^{-z}
\end{gathered}
$$

Cela permet en fait de calculer $G_{\text {out }}$ avec :

$$
\begin{aligned}
& \quad b=k_{0} \sqrt{X-1}, \quad a=z / \sqrt{X-1}, \quad c=\rho \\
& (-z>0 \text { si } z<0) . \\
& \text { Donc pour } G_{\text {out }}: \\
& \frac{\mathrm{e}^{j k_{0} \sqrt{z^{2}-(X-1) \rho^{2}}}}{4 \pi \sqrt{z^{2}-\rho^{2}(X-1)}} \quad z>0 \frac{z}{\sqrt{X-1}}>\rho \\
& \frac{\mathrm{e}^{j k_{0} \sqrt{z^{2}-(x-1) \rho^{2}}}}{4 \pi \sqrt{z^{2}-(X-1) \rho^{2}}} \quad z<0 \frac{-z}{\sqrt{X-1}}>\rho
\end{aligned}
$$

$\frac{-j \mathrm{e}^{-k_{n} \sqrt{(x-1) \rho^{2}-z^{2}}}}{4 \pi \sqrt{(X-1) \rho^{2}-z^{2}}} \quad z>0 \frac{z}{\sqrt{X-1}}<\rho$

$-j \frac{\mathrm{e}^{-k_{0} \sqrt{(X-1) \rho^{2}-z^{2}}}}{4 \pi \sqrt{(X-1) \rho^{2}-z^{2}}} \quad z<0 \frac{-z}{\sqrt{X-1}}<\rho$.

Donc pour $G_{\text {out }}$ :

$\frac{\mathrm{e}^{j k_{0} \sqrt{z^{2}-\rho^{2}(X-1)}}}{4 \pi \sqrt{z^{2}-\rho^{2}(X-1)}} \quad z^{2}>\rho^{2}(X-1)$

$\frac{-j \mathrm{e}^{-k_{0} \sqrt{(X-1) \rho^{2}-z^{2}}}}{4 \pi \sqrt{(X-1) \rho^{2}-z^{2}}} \quad z^{2}<\rho^{2}(X-1)$.

Pour $G_{\text {in }}=G_{\text {out }}^{*}$ :

$\frac{\mathrm{e}^{-j k_{0} \sqrt{z^{2}-\rho^{2}(X-1)}}}{4 \pi \sqrt{z^{2}-\rho^{2}(X-1)}} \quad z^{2}>\rho^{2}(X-1)$

$\frac{j \mathrm{e}^{-k_{0} \sqrt{(X-1) \rho^{2}-z^{2}}}}{4 \pi \sqrt{\rho^{2}(X-1)-z^{2}}} \quad z^{2}<\rho^{2}(X-1)$.

Les formules (III.9), (III.10) et (III.11) sont en accord avec celles données par Lee et Papas ( $3^{\mathrm{e}}$ article), mais pas la dernière formule.

Le changement $k_{0} \rightarrow-k_{0}$ invoqué y est d'ailleurs inexpliqué. 
IV. Puissance rayonnée. - On a vu dans les cas :

biaxe : $\quad \boldsymbol{\Gamma}_{\text {in }}=\boldsymbol{\Gamma}_{\text {out }}^{*} \rightarrow \mathscr{G}_{\text {in }}=-\mathscr{G}_{\text {out }}^{*}$

uniaxe : $\quad \Gamma_{\text {in }}=\Gamma_{\text {out }}^{*} \rightarrow \mathscr{G}_{\text {in }}=-\mathscr{G}_{\text {out }}^{*}$.

La lettre $\mathscr{G}$ est maintenant réservée à la véritable fonction de Green. Or :

$P_{\text {irr }}=-\frac{1}{4} \operatorname{Re} \int_{\text {espace }} \mathbf{J}^{*} .\left(\mathbf{E}_{\text {out }}-\mathbf{E}_{\text {in }}\right) \underline{\mathrm{d} \mathbf{r}}$

$P_{\text {rev }}=-\frac{1}{4} \operatorname{Re} \int_{\text {espace }} \mathbf{J}^{*} .\left(\mathbf{E}_{\text {out }}+\mathbf{E}_{\text {in }}\right) \underline{\mathrm{d} \mathbf{r}}$.

Pour un dipôle de moment $\mathbf{p}$, on obtient :

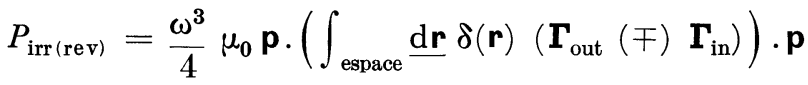

dans les deux cas, avec :

$$
\boldsymbol{\Gamma}_{\text {out }}=\boldsymbol{\Gamma}_{\text {out }}\left(\mathbf{B}_{0}\right), \quad \boldsymbol{\Gamma}_{\text {in }}=\boldsymbol{\Gamma}_{\text {in }}\left(-\mathbf{B}_{0}\right)
$$

dans le cas biaxe,

sans confusion possible dans le cas uniaxe.

Comme avec ces notations on a toujours :

$$
\boldsymbol{\Gamma}_{\text {out }}=\boldsymbol{\Gamma}_{\text {in }}^{*}
$$

on a encore :

$$
\begin{gathered}
P_{\text {irr }}=\frac{\omega^{3} \mu_{0}}{2} \mathbf{p} \cdot\left(\int \underline{\mathrm{d} \mathbf{r}} \delta(\mathbf{r}) \operatorname{Im}\left(\boldsymbol{\Gamma}_{\text {out }}\right)\right) \cdot \mathbf{p}=\underset{\begin{array}{c}
\text { totale } \\
\text { classique }
\end{array}}{P} \\
P_{\text {rev }}=0 .
\end{gathered}
$$

Ces démonstrations ont été faites en appliquant de très légères propriétés de symétrie aux intégrales définissant les éléments entrant et sortant mais uniquement après leur définition par la condition de rayonnement.

Note 1. - La nullité de $P_{\text {rev }}$ peut cependant être obtenue par élimination de termes infinis (cas de résonance) mais demeure. Les démonstrations ont été faites sur les intégrants.

Note 2. - Les propriétés ainsi démontrées sont vraies pour $X=2$ (cas $\varepsilon_{3}=-1$ ) où s'est placé Staras pour effectuer le calcul de la fonction de Green dans le cas uniaxe. Les formules (III.9) à (III.12) sont applicables et on trouve :

$$
\begin{aligned}
& G_{1 \mathrm{out}}-G_{1 \mathrm{in}}=j \frac{\sin k_{0} \sqrt{z^{2}-\rho^{2}}}{2 \pi \sqrt{z^{2}-\rho^{2}}}|z|>\rho \\
& =-j \frac{\mathrm{e}^{-k_{0} \sqrt{\rho^{2}-z^{2}}}}{2 \pi \sqrt{\rho-z^{2}}} \quad|z|<\rho
\end{aligned}
$$

ce qui est cohérent avec les résultats de Staras (Staras utilise la variation $\mathrm{e}^{j \omega t}$, Papas et Lee $\mathrm{e}^{-j \omega t}$ ).

$G_{1 \text { out }}-G_{1 \text { in }}$ et par là $\mathbf{E}_{\text {out }}-\mathbf{E}_{\text {in }}$ ont bien une singularité sur le cône de résonance.

V. Conclusion. - Les champs «entrant » et « sortant » $\mathbf{E}_{\text {in }}$ et $\mathbf{E}_{\text {out }}$ vérifient pour le courant vrai dans l'émetteur (compte tenu de la remarque (I.14.15)) :

$$
\mathbf{E}_{\text {in }}\left(-t,-\mathbf{B}_{0}\right)=\mathbf{E}_{\text {out }}^{*}\left(t, \mathbf{B}_{0}\right)
$$

et les fonctions de Green :

$$
\mathscr{G}_{\text {in }}^{*}\left(t, \mathbf{B}_{0}\right)=-\mathscr{G}_{\text {out }}^{*}\left(-t,-\mathbf{B}_{\mathbf{0}}\right)
$$

dans un plasma avec champ magnétique. Dans le cas où ce champ est infini, les mêmes relations sont valables où n'intervient pas l'élément $\mathbf{B}_{0}$.

- Le partage $P=P_{\text {irr }}+P_{\text {rev }}$ conduit à $P_{\text {rev }}=0$.

- L'introduction de collisions dans la théorie, si petite que soit leur importance, supprime le cône singulier. Cependant, cela ne suffit pas à supprimer les infinis de puissance rayonnée dans le cas de la représentation du courant par la fonction $\delta$ (cf. WeilLafon [5]). Des dimensions finies imposées à l'émetteur petit mais non ponctuel permettent de pallier cet inconvénient (cf. [5]).

- $\mathbf{E}_{\text {in }}-\mathbf{E}_{\text {out }}$, solution de l'équation homogène, a une singularité sur le cône de résonance.

- Staras partant d'une équation d'onde homogène en coordonnées sphériques la singularise à l'origine et ne peut de ce fait infirmer l'assertion selon laquelle cette équation admettrait seulement des solutions sans singularités.

- Dans le cas sans collisions, comme le disent Lee et Papas, on ne peut pas appliquer le théorème d'Ostrogradsky, donc calculer le flux du vecteur de Poynting à travers une grande sphère comme égal à l'expression traditionnelle de la puissance à cause des singularités sur le cône de résonance. Cet handicap disparaît, comme le cône, avec l'introduction de collisions.

- Il est enfin vrai que dans le cas (en l'absence de collisions) où le cône de résonance existe, on ne peut changer l'ordre des intégrations dans le calcul de la puissance rayonnée; en effet, l'une des façons d'intégrer conduisant à une valeur infinie, l'intégrale multiple formée en unissant toutes les intégrations n'est pas définie. On ne peut évidemment pas lui appliquer le théorème de Fubini, ce à quoi revient la modification de l'ordre des intégrations. Mais on a cependant $\boldsymbol{\Gamma}_{\text {in }}=\boldsymbol{\Gamma}_{\text {out }}^{*}($ notation (IV .6)) pour tout $\mathbf{r}$, et $P_{\text {rev }}=0$. 


\title{
BIBLIOGRAPHIE
}

[1] LEE et PAPAS, Radiation resistance and irreversible power of antennas in gyroelectric media, IEEE Trans. Ant. Prop., A.P. 13, 5, 834-835 ; Irreversible power and radiation resistance of antennae in anisotropic ionized gases, Radio Science J. Res., NBS 69D, 10, 1313-1320 ; A further explanation of the new theory of antenna radiation with particular reference to uniaxial media, Radio Science J. Res., sept. 66, 1, 9, 1020-1023.

[2] STARAS, The "infinity catastrophe" associated with radiation in magnetoionic media, Radio Science J. Res., sept. 66, 1, 9, 1013-1020.
[3] WALSH (D.) et WEIL (H.), Irreversible power and radiation resistance of antenna in magnetoionic media, Radio Science J. Res., sept. 66, 1, 9, 10251027.

[4] KOGEINIK (H.), On electromagnetic radiation in magnetoionic media, J. Res. Nat. Bur. Stand., sept.-oct. 1960, 64 D, 5 .

[5] LAfFON (J. P.) et WEIL (H.), Impedance of "cylindrical" antennas in magnetoplasma under conditions of refractive index resonance. A paraître.

[6] LAFON (J. P.), Condition de rayonnement-Condition d'antirayonnement, 1968.

\section{OBSERVATION SIMULTANÉE DE DEUX GROUPES DE RAIES D'UN SPEGTRE DE MASSE}

\author{
Par M. BEGOURT, \\ Laboratoire de Physique des Milieux Ionisés, École Polytechnique, Équipe de Recherche associée au G.N.R.S.
}

(Reçu le 9 avril 1968.)

Résumé. - Nous décrivons ici un générateur de tension avec ses circuits associés qui permet de balayer deux raies ou groupes de raies quelconques sur un spectromètre de masse, et de visualiser l'évolution de leurs amplitudes en fonction du temps. Le signal de sortie de la base de temps est la somme d'une tension continue d'une tension en dent de scie à la fréquence $f$, et avec la même phase, d'une tension carrée à la fréquence $f / 2$.

Abstract. - A special power supply and its associated circuits are described. The output voltage is applied to a mass spectrometer in order to sweep two lines or any group of lines and to depict the variations of the amplitudes versus time on an oscilloscope. The waveshape of the time base is the sum of a direct current voltage, a sawtooth of frequency $f$ and with the same phase, and a square wave of frequency $t / 2$.

I. Introduction. - Un analyseur de spectre permet d'obtenir un déroulement continu du spectre de masse, entre des limites fixées au préalable. Ce déroulement est d'autant plus lent que le spectre à observer est plus large, et s'il permet parfaitement l'enregistrement graphique, il est très difficile de comparer sur un oscilloscope deux raies ou groupes de raies, de masses et d'intensités très différentes.

Pour remédier à cet inconvénient, nous avons réalisé une base de temps qui, associée à un analyseur « Veeco GA-4 » et à un oscilloscope « Tektronix 561-A», permet d'observer deux parties quelconques du spectre, de largeur variable, sur chacune des traces d'un tiroir $3 \mathrm{~A} 3$, et de comparer simultanément les amplitudes respectives des masses, même si celles-ci sont très différentes en choisissant la sensibilité de chacune des voies.

II. Fonctionnement de l'analyseur « Veeco GA-4 ». Le tube analyseur, qui est l'élément sensible du spectromètre de masse, requiert une alimentation haute tension variable, un amplificateur électrométrique à liaison directe et un organe de lecture du type oscilloscope ou enregistreur.

Pour balayer tout le spectre, il faut, quel que soit le mode de fonctionnement utilisé : manuel ou automatique, appliquer à l'amplificateur qui commande le tube un signal variable de 0 à $150 \mathrm{~V}$. Par suite de 